\title{
Charmonia and bottomonia at finite temperature on large quenched lattice
}

\author{
Hiroshi Ohno* \\ Center for Computational Sciences, University of Tsukuba, Tsukuba, Ibaraki 305-8577, Japan \\ Physics Department, Brookhaven National Laboratory, Upton, NY 11973, USA \\ E-mail: hohnodccs.tsukuba.ac.jp
}

\begin{abstract}
We show our updated study on charmonia and bottomonia at finite temperature with quenched ensembles on large and fine isotropic lattices. Simulations have been performed by using the standard plaquette gauge and the $O(a)$-improved Wilson fermion actions with quark masses for both charm and bottom. We tested a stochastic method to reconstruct spectral functions with some mock data and also applied it to our lattice data. We investigated temperature dependence of the charmonium and bottomonium spectral functions in the vector channel at temperatures in a range between about $0.73 T_{c}$ and $2.2 T_{c}$. We found that $J / \psi$ seems to melt at $T<1.5 T_{c}$ while $\Upsilon$ may still exist $T>1.5 T_{c}$
\end{abstract}

The 33rd International Symposium on Lattice Field Theory

14 -18 July 2015

Kobe International Conference Center, Kobe, Japan

\footnotetext{
* Speaker.
} 


\section{Introduction}

Suppression of quarkonium yields is one of important signals for formation of the quark-gluon plasma [1] in relativistic heavy ion collisions. In fact, several experimental results indicating suppression of charmonia [2, 3, 4, 5, 6] and bottomonia [7] have been reported already and theoretical understanding of in-medium properties of quarkonia, especially the melting temperatures, plays an important role to explain the experimental results.

To this end the quarkonium spectral function (SPF), which has all information about quarkonia in hot medium, is an important quantity. However, what one can directly calculate in lattice QCD simulations is only the Euclidean time correlation function, which is related to the SPF through a Laplace transform, and an inverse problem needs to be solved to get the SPF. Here typical number of data points of the correlation function obtained from recent lattice QCD simulations is $O(10)$ while the SPF is a continuous function. Therefore, getting the SPF from the correlation function is an ill-posed problem and it is known that a simple $\chi^{2}$-fitting does not work in general. To overcome this the maximum entropy method (MEM) [8] is one of the most commonly used methods.

By using the MEM charmonim SPFs have been studied already in quenched QCD [9, 10] and in 2-flavor QCD [11]. However, the recent study [10] and old studies [9 11] have different conclusions on the melting temperature of $J / \Psi$. Recently bottomonium SPFs have also been investigated in NRQCD [12] and survival of S-wave states up to $2 T_{c}$ and melting of P-wave states above $T_{c}$, where $T_{c}$ is the critical temperature, have been indicated. On the other hand, very recently a bottomonium study also in the NRQCD framework but using a novel Bayesian analysis [13] suggested that a P-wave state still survives at high temperature. Since quarkonium melting temperatures are still not conclusive within different lattice setup and different reconstruction methods of SPFs, a further study with more precise data and making comparison among several reconstruction methods of SPFs is needed to crosscheck previous results and estimate systematic uncertainties.

In this study we show our recent study on reconstruction of the charmonium and bottomonium SPFs as an update of our previous studies [15, 16] about quarkonia at finite temperature on large quenched lattices. Instead of the MEM we introduce a stochastic method to reconstruct SPFs, which is briefly reviewed in the next section. In Sec. 3 a mock-data test of the stochastic method and its application to lattice data are shown. Finally, Sec. 4 gives the summary and our future plan.

\section{A stochastic method to reconstruct spectral functions}

Lattice simulations obtain the Euclidean time correlation function $G(\tau)$ directly while the SPF $\rho(\omega)$ is given only from the following relation:

$$
G(\tau)=\int \frac{d \omega}{2 \pi} \rho(\omega) K(\omega, \tau)
$$

where $K(\omega, \tau) \equiv \cosh (\omega(\tau-1 / 2 T)) / \sinh (\omega / 2 T)$ is an integral kernel at a given temperature $T=1 / N_{\tau} a$, where $N_{\tau}$ and $a$ are the temporal extent and the lattice spacing, respectively. Our goal is to reconstruct $\rho(\omega)$ from a given data set of $G(\tau)$. In rest of this section we briefly review a stochastic method based on a Bayesian inference to reconstruct SPFs, which is called the Stochastic Analytical Inference (SAI) [17. 


\subsection{Bayesian statistical inference}

Let us first introduce a smooth mapping $\phi: \mathbb{R} \mapsto\left[0, x_{\max }\right]$, which takes the frequency of the SPF onto a new variable $x$ as $x \equiv \phi(\omega)=\int_{-\infty}^{\omega} \frac{d \omega^{\prime}}{2 \pi} D\left(\omega^{\prime}\right) K\left(\omega^{\prime}, \tau_{0}\right)$, where $\tau_{0}$ is a reference imaginary time for normalization, $x_{\max }=\phi(\infty)$ and $D(\omega)$ is a positive definite function called a default model (DM), which contains prior information about the SPF. By taking a change of variable $\omega \rightarrow x$, (2.1) can be written as $G(\tau)=\int_{0}^{x_{\max }} d x n(x) \tilde{K}(x, \tau)$, where $n(x) \equiv \rho\left(\phi^{-1}(x)\right) / D\left(\phi^{-1}(x)\right)$ and $\tilde{K}(x, \tau) \equiv$ $K\left(\phi^{-1}(x), \tau\right) / K\left(\phi^{-1}(x), \tau_{0}\right)$. Then we get a normalization condition of $n(x)$ as

$$
\int_{0}^{x_{\max }} d x n(x)=G\left(\tau_{0}\right) .
$$

Next, let us consider how to get the most likely $n(x)$ for given prior knowledge $D(\omega)$. Suppose we have $N$ points of Monte Carlo averaged data $\bar{G}$ with their covariance matrix $C_{\tau, \tau^{\prime}} \equiv$ $\overline{G(\tau) G\left(\tau^{\prime}\right)}-\bar{G}(\tau) \bar{G}\left(\tau^{\prime}\right)$, the Bayes' theorem tells us a relation $P[n \mid \bar{G}]=P[\bar{G} \mid n] P[n] / P[\bar{G}]$, where $P[n \mid \bar{G}]$ denotes the posterior probability of $n(x)$ for given data $\bar{G}(\tau), P[\bar{G} \mid n]$ the likelihood function, $P[n]$ the prior probability of $n(x)$ and $P[\bar{G}]$ is called the evidence, which just plays a role of normalization for $P[n \mid \bar{G}]$. Here the likelihood function is defined as $P[\bar{G} \mid n]=e^{-\chi^{2}[n] / 2 \alpha} / Z^{\prime}$, where $\alpha>0$ is a regularization parameter controlling the contribution of $P[\bar{G} \mid n]$ relative to $P[n]$, $\chi^{2}[n] \equiv \sum_{\tau, \tau^{\prime}}^{N}(G(\tau)-\bar{G}(\tau)) C_{\tau, \tau^{\prime}}^{-1}\left(G\left(\tau^{\prime}\right)-\bar{G}\left(\tau^{\prime}\right)\right)$ and $Z^{\prime}=\int \mathscr{D} \bar{G} e^{-\chi^{2}[n] / 2 \alpha}=(2 \pi \alpha)^{N / 2} \sqrt{\operatorname{det} C}$. Positivity of $n(x)$ and the normalization condition (2.2) can be set to the prior probability as $P[n]=\Theta[n] \delta\left(\int_{0}^{x_{\max }} d x n(x)-G\left(\tau_{0}\right)\right)$. By definition $P[\bar{G}]=\int \mathscr{D}^{\prime} n e^{-\chi^{2}[n] / 2 \alpha} / Z^{\prime}=Z / Z^{\prime}$, where $\mathscr{D}^{\prime} n \equiv \mathscr{D} n P[n]$. As a consequence,

$$
P[n \mid \bar{G}]=\frac{1}{Z} \Theta[n] \delta\left(\int_{0}^{x_{\max }} d x n(x)-G\left(\tau_{0}\right)\right) e^{-\chi^{2}[n] / 2 \alpha} .
$$

Thus, by taking average over all possible $n(x)$ weighted by $P[n \mid \bar{G}]$, we get

$$
\langle n(x)\rangle_{\alpha}=\int \mathscr{D}^{\prime} n n(x) e^{-\chi^{2}[n] / 2 \alpha},
$$

which is the most likely $n(x)$ for given $\alpha$. In the limit $\alpha \rightarrow 0, P[n \mid \bar{G}]$ dominates relative to $P[n]$, which is equivalent to the $\chi^{2}$-fitting. On the other hand, for $\alpha \rightarrow \infty$ the SPF converges to the DM.

Since the regularization parameter $\alpha$ is introduced by hand, final SPFs must be independent of $\alpha$. There have been several ways to eliminate $\alpha$ and here we consider following two procedures:

1. Calculating the posterior probability of $\alpha$,

$$
P[\alpha \mid \bar{G}]=P[\alpha] \int \mathscr{D} n \frac{P[\bar{G} \mid n, \alpha] P[n \mid \alpha]}{P[\bar{G}]} \propto P[\alpha] \alpha^{-N / 2} Z(\alpha)
$$

by using the Bayes' theorem again, where $P[\alpha]$ is the prior probability of $\alpha$ and is usually taken to be $1 / \alpha$, then choosing $\alpha=\alpha_{\text {peak }}$ at a peak location of $P[\alpha \mid \bar{G}]$ or taking average of $\langle n(x)\rangle_{\alpha}$ over $\alpha$ weighted by $P[\alpha \mid \bar{G}]$.

2. Choosing $\alpha=\alpha_{\text {kink }}$ at a kink of a $\ln \alpha-\ln \left(\left\langle\chi^{2}\right\rangle_{\alpha} / N\right)$ curve [18].

Note that it has been shown in [18] that a mean field treatment of $n(x)$ in the SAI turns out to be equivalent to the MEM. 


\subsection{Monte Carlo evaluation}

To compute (2.4) the integral with respect to $n(x)$ needs to be evaluated numerically. This can be done by an importance sampling procedure. To do so, $n(x)$ is represented by a superposition of a fixed number $M$ of delta functions with residues $r_{i} \geq 0$ and coordinates $0 \leq x_{i} \leq x_{\max }$ as $n(x)=\sum_{i=1}^{M} r_{i} \delta\left(x-x_{i}\right)$. In this case, the normalization condition (2.2) turns to be

$$
\sum_{i=1}^{M} r_{i}=G\left(\tau_{0}\right)
$$

There are only two types of the update schemes conserving the normalization condition (2.6) and satisfying detailed balance for a fixed $M$ as follows :

1. Shifting delta functions : $x_{i} \rightarrow x_{i}^{\prime}$

2. Changing residues of delta functions by keeping (2.6) $: \sum_{i} r_{i}=\sum_{i} r_{i}^{\prime}$

An update is accepted by the probability $P=\min \left\{1, e^{-\Delta \chi^{2} / 2 \alpha}\right\}$, where $\Delta \chi^{2}$ is the difference of $\chi^{2}$ values between configurations before and after an attempt.

\section{Numerical results}

\subsection{Mock-data test}

To see how the SAI works first we applied it to a mock data. A mock SPF considered here consists of a resonance peak and a continuum defined as

$$
\begin{aligned}
\rho(\omega) & =\rho_{\text {res }}(\omega)+\rho_{\text {cont }}(\omega), \\
\rho_{\text {res }}(\omega) & =c_{\text {res }} \frac{\Gamma\left(\omega ; \omega_{0}, \gamma_{0}\right) M}{\left(\omega^{2}-M^{2}\right)^{2}+M^{2} \Gamma^{2}\left(\omega ; \omega_{0}, \gamma_{0}\right)} \omega^{2}, \quad \Gamma\left(\omega ; \omega_{0}, \gamma_{0}\right)=\Theta\left(\omega-\omega_{0}\right) \gamma_{0}\left(1-\frac{\omega_{0}^{2}}{\omega^{2}}\right)^{5}, \\
\rho_{\text {cont }}(\omega) & =c_{\text {cont }} \frac{3}{4 \pi} \Theta\left(\omega^{2}-4 m^{2}\right) \omega^{2} \tanh \left(\frac{\omega}{4 T}\right) \sqrt{1-\left(\frac{2 m}{\omega}\right)^{2}}\left[2+\left(\frac{2 m}{\omega}\right)^{2}\right],
\end{aligned}
$$

where we chose $\left(c_{\text {res }}, \omega_{0}, \gamma_{0}, M\right)=(2,0.2,0.2,0.5)$ and $\left(c_{\text {cont }}, m\right)=(20,0.1)$. The mock correlation function was created in a range $1 \leq \tau / a \leq N_{\tau} / 2$ with $N_{\tau}=48$ and by adding a Gaussian random noise to a value computed by (2.1) at each $\tau$, where variance of the noise was defined by $\sigma(\tau)=$ $\varepsilon G(\tau) \tau$ and $\varepsilon$ was set to $10^{-4}$. From 200 samples a full covariance matrix was computed.

For the SAI we set the number of delta functions $M$ to 10000 . After performing $50000 \times M$ Monte Carlo updates as thermalization, we sampled 100 possible spectra at every $100 \times M$ updates at each $\alpha$. We adopted the parallel tempering to compute spectra at 420 equally-divided $\ln \alpha$ values in a range $10^{-6} \leq \alpha \leq 10^{14}$ simultaneously. The reference imaginary time $\tau_{0} / a$ was set to 1 . On the r.h.s of Fig. 1 we show output SPFs for three different choices of $\alpha$ : SPFs at $\alpha_{\text {peak }}, \alpha_{\text {kink }}$ and averaged over $\alpha$ weighted by $P[\alpha \mid \bar{G}]$. Here the DM has a form $D(\omega)=c \omega^{2}$, where the overall factor $c$ is irrelevant since it just plays a role to fix $x_{\max }$. The kink of $\left\langle\chi^{2}\right\rangle_{\alpha} / N$ curve shown on the 1.h.s of Fig. 1 1 was defined by a peak location of $d^{2} \ln \left(\left\langle\chi^{2}\right\rangle_{\alpha} / N\right) / d(\ln \alpha)^{2}$. Since the partition function $Z(\alpha)$ is needed to compute $P[\alpha \mid \bar{G}]$ as shown in $(2.5)$, we computed the density of states 

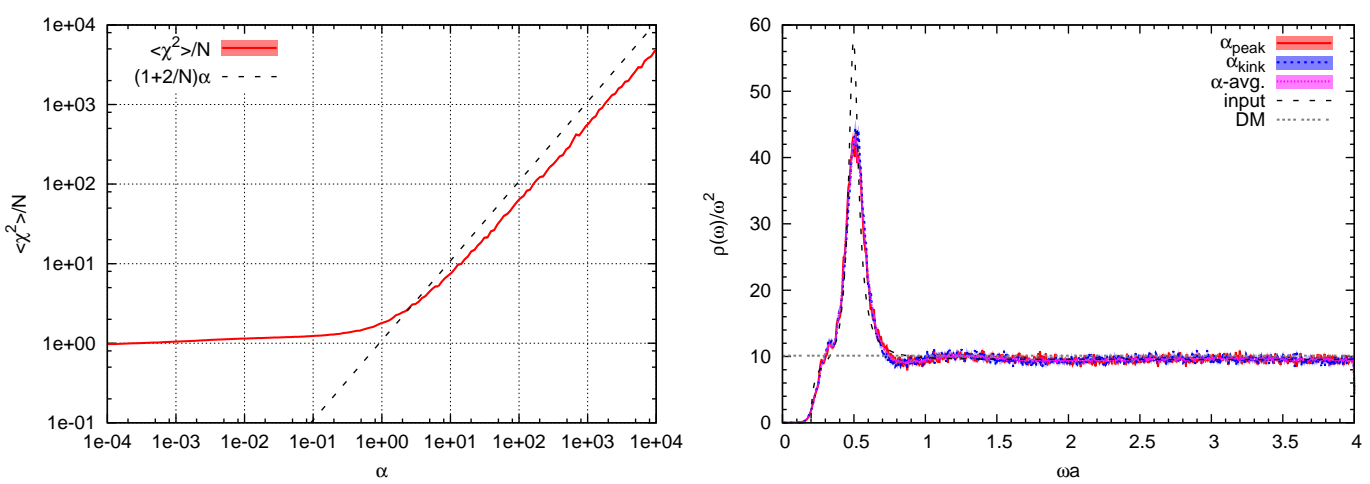

Figure 1: (Left) A log-log plot of $\left\langle\chi^{2}\right\rangle_{\alpha} / N$ as a function of $\alpha .(1+2 / N) \alpha$ is also shown by a dashed line as a guide to find a peak location of $P[\alpha \mid \bar{G}]$ (see text). (Right) SPFs given by the SAI. Results at $\alpha_{\text {peak }}$ and $\alpha_{\text {kink }}$ are shown by red and blue curves, respectively. A result averaged over $\alpha$ weighted by $P[\alpha \mid \bar{G}]$ is indicated by a magenta curve. Dashed and dotted curves correspond to the input mock SPF and the DM, respectively.

Table 1: Lattice setup.

\begin{tabular}{cccccc}
\hline \hline$\beta$ & $a[\mathrm{fm}]$ & $N_{\sigma}$ & $N_{\tau}$ & $T / T_{c}$ & \# of confs. \\
\hline 7.192 & 0.0188 & 96 & 48 & 0.73 & 259 \\
& & & 32 & 1.1 & 476 \\
& & & 28 & 1.25 & 336 \\
& & & 24 & 1.5 & 336 \\
& & & 16 & 2.2 & 239 \\
\hline \hline
\end{tabular}

for $n(x)$ with the Wang-Landau algorithm [19]. We also note that the peak location of $P[\alpha \mid \bar{G}]$ can be estimated by a relation $d P[\alpha \mid \bar{G}] /\left.d \alpha\right|_{\alpha=\alpha_{\text {peak }}} \propto\left\langle\chi^{2}\right\rangle_{\alpha_{\text {peak }}} / N-(1+2 / N) \alpha_{\text {peak }}=0$, which means that an intersection between $\left\langle\chi^{2}\right\rangle_{\alpha} / N$ curve and a dashed line on the 1.h.s of Fig. 10 should be located at $\alpha=\alpha_{\text {peak }}$. As it can be seen, there is quite small $\alpha$ dependence for the output SPFs. This is because that $\alpha_{\text {peak }}$ and $\alpha_{\text {kink }}$ are close to each other and $P[\alpha \mid \bar{G}]$ has a sharp peak at $\alpha=\alpha_{\text {peak }}$. The peak position of the input and output SPFs are almost the same while the peak hight and width of output SPFs is slightly smaller and broader than the input. On the other hand, the continuum is reproduced quite well.

\subsection{Charmonium and bottomonium SPFs in the vector channel}

Next we applied the SAI to our real lattice data. We performed our lattice simulations with the standard plaquette gauge and the $O(a)$-improved Wilson fermion actions on large and fine isotropic lattices as shown in Table 1 At temperatures in a range from $0.73 T_{c}$ to $2.2 T_{c}$ we generated gauge configurations with an over-relaxed pseudo-heatbath algorithm. After 2000 thermalization sweeps we measured the spatial component of vector current-current correlators $G(\tau) \equiv$ $\int d^{3} x\left\langle\sum_{i=1}^{3} J_{i}(\vec{x}, \tau) J_{i}(\overrightarrow{0}, 0)\right\rangle$, where $J_{i}(\vec{x}, \tau) \equiv \bar{q}(\vec{x}, \tau) \gamma_{i} q(\vec{x}, \tau)$, at every 500 sweeps with two different $\kappa$ values 0.13194 and 0.12257 , which were tuned so that corresponding vector meson masses 3.140 (3) and 9.574(3), are close to the experimental values of $J / \Psi$ and $\Upsilon$ masses, respectively 

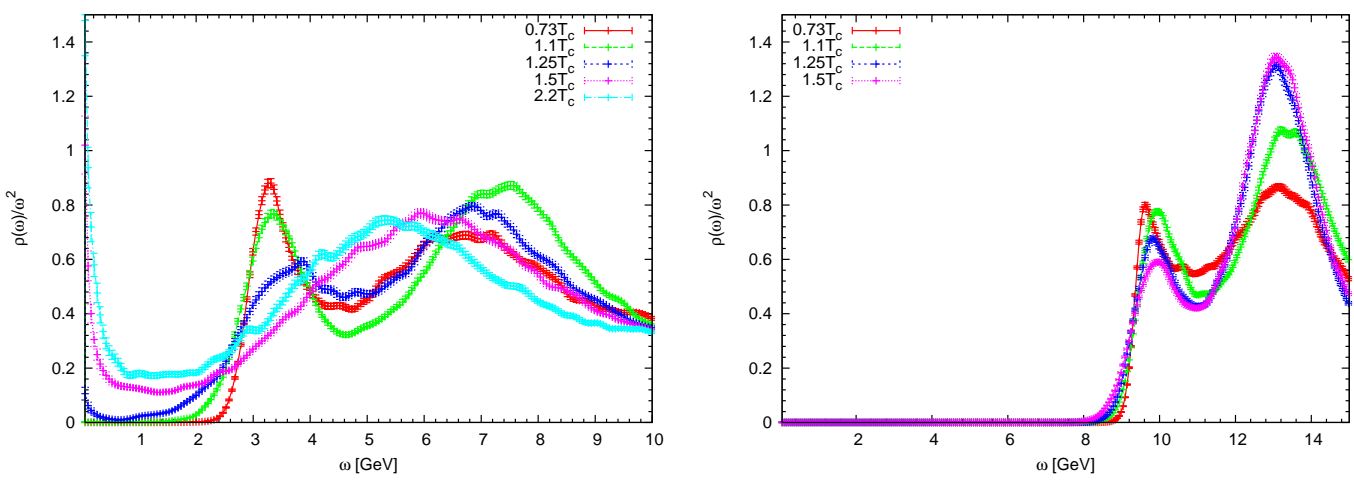

Figure 2: Temperature dependence of the SPF of the charmonium (left) and bottomonium (right) for the vector channel. Red, green, blue, magenta and light-blue curves correspond to the SPFs at $0.75,1.1,1.25$, 1.5 and $2.2 T_{c}$, respectively. The bottomonium SPF at $2.2 T_{c}$ is not shown since it is unstable for the current data quality.

[20]. The Sommer scale $r_{0} / a$ and the critical temperature $T_{c}$ have been given in [21] and we used $r_{0}=0.49 \mathrm{fm}$ to set our scale. In Fig. 2 the charmonium and bottomonium SPFs for the vector channel at each temperature are shown. The bottomonium result at $2.2 T_{c}$ is not shown since it is unstable for the current data quality. Here, as the first test, we just simply chose a DM $D(\omega) \propto K^{-1}\left(\omega, \tau_{0}\right)$,

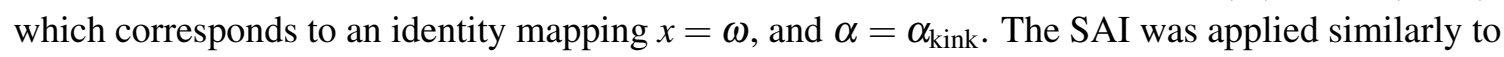
the mock-data test except that the number of updates for thermalization was set to $10000 \times M$. The 1.h.s of Fig. 2 2 suggested that the first peak corresponding to $J / \Psi$ has large temperature dependence and seems to disappear up to $1.5 T_{c}$. It also can be seen that a transport peak clearly appears around $\omega=0$ at temperatures above $1.25 T_{c}$. On the other hand, from the r.h.s of Fig. 2 , the $\Upsilon$ peak sitting around $\omega=10 \mathrm{GeV}$ looks stable against temperature change, which suggests survival of $\Upsilon$ up to our highest temperature $1.5 T_{c}$. There is no clear transport peak in the bottom case at temperatures investigated in this study so far.

\section{Summary}

We tested a stochastic method called the SAI to reconstruct SPFs with mock data having a resonance peak and a continuum. We found that the SAI has small dependence for choice of the regularization parameter $\alpha$ and can reproduce the location of the resonance peak and the continuum part quite well. Then we applied the SAI to our lattice data of the vector current-current correlators to reconstruct the charmonium and bottomonium SPF. We found that the $J / \Psi$ peak has large temperature dependence and seems to disappear up to $1.5 T_{c}$ while the $\Upsilon$ state is stable and may exist even at $1.5 T_{c}$. We also found that a transport peak appears above $T_{c}$ in the charm case but no transport peak was visible in the bottom case within our current setup so far.

Checking more systematic uncertainties with various DMs and by comparing our results to those with any other methods, e.g. the MEM and another stochastic method [22], are needed to conclude the quarkonium melting temperatures. Determining a transport coefficient, taking the continuum limit and extending the study to the finite momentum case are also our future plan. 


\section{Acknowledgments}

The author thank H.-T. Ding, O. Kaczmarek, S. Mukherjee and H.-T. Shu for fruitful discussions. The numerical calculations have been performed on the Bielefeld GPU cluster and the OCuLUS Cluster at The Paderborn Center for Parallel Computing in Germany.

\section{References}

[1] T. Matsui and H. Satz, Phys. Lett. B 178, 416 (1986).

[2] R. Arnaldi [NA60 Collaboration], Nucl. Phys. A 830, 345C (2009) [arXiv:0907.5004 [nucl-ex]].

[3] A. Adare et al. [PHENIX Collaboration], Phys. Rev. Lett. 101, 232301 (2008) [arXiv:0801.4020 [nucl-ex]].

[4] B. Abelev et al. [ALICE Collaboration], Phys. Rev. Lett. 109, 072301 (2012) [arXiv:1202.1383 [hep-ex]].

[5] G. Aad et al. [ATLAS Collaboration], Phys. Lett. B 697, 294 (2011) [arXiv:1012.5419 [hep-ex]].

[6] S. Chatrchyan et al. [CMS Collaboration], JHEP 1205, 063 (2012) [arXiv:1201.5069 [nucl-ex]].

[7] S. Chatrchyan et al. [CMS Collaboration], Phys. Rev. Lett. 109, 222301 (2012) [arXiv:1208.2826 [nucl-ex]].

[8] M. Asakawa, T. Hatsuda and Y. Nakahara, Prog. Part. Nucl. Phys. 46, 459 (2001) [hep-lat/0011040].

[9] A. Jakovac, P. Petreczky, K. Petrov and A. Velytsky, Phys. Rev. D 75, 014506 (2007) [hep-lat/0611017].

[10] H. T. Ding, A. Francis, O. Kaczmarek, F. Karsch, H. Satz and W. Soeldner, Phys. Rev. D 86, 014509 (2012) [arXiv:1204.4945 [hep-lat]].

[11] G. Aarts, C. Allton, M. B. Oktay, M. Peardon and J. -I. Skullerud, Phys. Rev. D 76, 094513 (2007) [arXiv:0705.2198 [hep-lat]].

[12] G. Aarts, C. Allton, T. Harris, S. Kim, M. P. Lombardo, S. M. Ryan and J. I. Skullerud, JHEP 1407, 097 (2014) [arXiv:1402.6210 [hep-lat]].

[13] Y. Burnier and A. Rothkopf, Phys. Rev. Lett. 111, 182003 (2013) [arXiv:1307.6106 [hep-lat]].

[14] S. Kim, P. Petreczky and A. Rothkopf, Phys. Rev. D 91, 054511 (2015) [arXiv:1409.3630 [hep-lat]].

[15] H. Ohno, PoS LATTICE 2013, 172 (2013) [arXiv:1311.4565 [hep-lat]].

[16] H. Ohno, H.-T. Ding and O. Kaczmarek, PoS LATTICE 2014, 219 (2014) [arXiv:1412.6594 [hep-lat]].

[17] Sebastian Fuchs, Thomas Pruschke and Mark Jarrell, Phys. Rev. E 81, 056701 (2010) [arXiv:0912.5204 [cond-mat.str-el]].

[18] K. S. D. Beach, [arXiv:cond-mat/0403055 [cond-mat.str-el]].

[19] Fugao Wang and D. P. Landau, Phys. Rev. E 64, 056101 (2001) [arXiv:cond-mat/0107006 [cond-mat.stat-mech]].

[20] J. Beringer et al. [Particle Data Group Collaboration], Phys. Rev. D 86, 010001 (2012).

[21] A. Francis, O. Kaczmarek, M. Laine, T. Neuhaus and H. Ohno, Phys. Rev. D 91, no. 9, 096002 (2015) [arXiv:1503.05652 [hep-lat]].

[22] H. T. Shu, H. T. Ding, O. Kaczmarek, S. Mukherjee and H. Ohno, arXiv:1510.02901 [hep-lat]. 